\title{
Indicadores de Sostenibilidad de vivienda asequible para la ciudad de Cuenca-Ecuador
}

\section{Affordable Housing Sustainability Indicators for the city of Cuenca-}

\author{
Ecuador
}

1 José Alvarado Orbe

Universidad Católica de Cuenca, Maestría en Construcciones con mención en

Administración de la Construcción Sustentable, Cuenca, Ecuador,

jose.alvarado.13@est.ucacue.edu.ec

2 Juan Felipe Quesada Molina

Universidad Católica de Cuenca, Maestría en Construcciones con mención en

Administración de la Construcción Sustentable, Cuenca, Ecuador,

felipe.quesada@ucacue.edu.ec

3 Enma Alexandra Espinosa Iñiguez

https://orcid.org/0000-0002-3770-3688

Universidad Católica de Cuenca, Carrera de Arquitectura, Cuenca, Ecuador,

eespinosai@ucacue.edu.ec

\begin{tabular}{r}
\hline Artículo de Investigación Científica y Tecnológica \\
Enviado: $08 / 12 / 2021$ \\
Revisado: $23 / 12 / 2021$ \\
Aceptado: $17 / 01 / 2022$ \\
Publicado:05/03/2022 \\
DOI: $\underline{\text { https://doi.org/10.33262/concienciadigital.v5i1.2.2086 }}$
\end{tabular}

Cítese: $\quad$ lvarado Orbe, J., Quesada Molina, J. F., \& Espinosa Iñiguez, E. A. (2022). Indicadores de Sostenibilidad de vivienda asequible para la ciudad de Cuenca-Ecuador.

ConcienciaDigital, 5(1.2), 66-85. https://doi.org/10.33262/concienciadigital.v5i1.2.2086

CONCIENCIA DIGITAL, es una Revista Multidisciplinar, Trimestral, que se publicará en soporte electrónico tiene como misión contribuir a la formación de profesionales competentes con visión humanística y crítica que sean capaces de exponer sus resultados investigativos y científicos en la misma medida que se promueva mediante su intervención cambios positivos en la sociedad. https://concienciadigital.org

La revista es editada por la Editorial Ciencia Digital (Editorial de prestigio registrada en la Cámara Ecuatoriana de Libro con No de Afiliación 663) www.celibro.org.ec 
Palabras

claves: Palabras

Clave:

arquitectura

urbana,

indicadores,

recursos,

sostenibilidad

urbana,

vivienda

asequible

\section{Keywords:}

Urban

Architecture,

Indicators,

Resources,

Urban

Sustainability,

Affordable

Housing.

\section{Resumen}

Introducción: En el presente trabajo se analiza a la sostenibilidad urbana la cual es una visión reciente que combina la protección del medio ambiente, participación social y crecimiento económico equitativo con la finalidad de solucionar los muchos problemas sociales que existen y al mismo tiempo incrementar la calidad de vida de las personas, por otra parte al hablar de calidad podemos hacer referencia a la vivienda asequible la cual es un tipo de vivienda que combina todas las comodidades de una vivienda normal con un manejo responsable de los recurso ambientales, económicos y sociales. Objetivo principal: diseñar un grupo de indicadores de sostenibilidad urbana enfocados en la vivienda asequible para la Ciudad de Cuenca-Ecuador. Metodología: enfoque caritativo mediante una revisión bibliográfica de trabajos y normativas internacionales y nacionales que regulen el diseño de estos indicadores. Resultados: Finalmente se obtuvo como resultado que la tasa de empleo de la ciudad es del 58.65\%, existe 1 vehículo de recolección de basura por cada 15432 habitantes y solo el $28.98 \%$ del salario promedio mensual de los ciudadanos de cuenca se destina a pago de servicios básicos

\section{Abstract}

Introduction: This paper analyzes urban sustainability, which is a recent vision that combines environmental protection, social participation, and equitable economic growth to solve the many social problems that exist and at the same time increase quality. of people's lives, on the other hand when talking about quality we can refer to affordable housing which is a type of housing that combines all the comforts of a normal home with a responsible management of environmental, economic, and social resources. Main objective: to design a group of urban sustainability indicators focused on affordable housing for the City of Cuenca-Ecuador. Methodology: charitable approach through a bibliographic review of works and international and national regulations that regulate the design of these indicators. Results: Finally, it was obtained as a result that the employment rate of the city is $58.65 \%$, there is one garbage collection vehicle for every 15,432 inhabitants and only $28.98 \%$ of the average monthly salary of the citizens of Cuenca is destined to payment of basic services 


\section{Introducción}

En la última década el nerviosismo por los cambios negativos del medio ambiente se ha visto incrementado, debido a ello en 1972 en el marco de la Conferencia acerca del Medio Humano de la Organización de las Naciones Unidas (ONU), nace la preocupación por el cuidado del medio ambiente y búsqueda de la sostenibilidad convirtiéndose en elementos principales para conseguir una mejora de la calidad de vida de los seres humanos (Salas et al., 2016).

Por lo que para conseguir esta mejora se necesita de una sostenibilidad la cual está dirigida a satisfacer las necesidades actuales sin complicar la capacidad de las próximas generaciones de satisfacer las suyas. Además de garantizar el equilibrio entre cuidado del medio ambiente, desarrollo económico y prosperidad social, colocando en duda la posibilidad del crecimiento urbano en todas las escalas y trazando una necesidad de cambio con respecto a esta situación (Báez et al., 2019). Por otra parte, en los países latinoamericanos, el mayor problema de desarrollo urbano se centraliza en la vivienda social o vivienda para ciudadanos de bajos recursos económicos. Pero, os fondos que los estados destinan a mencionada infraestructura no son proporcionales a la dimensión del problema (Álvarez, 2016). Por lo que las distintas soluciones arquitectónicas planteadas por los Estados no poseen las siguientes condiciones, que son de gran importancia para conseguir un entorno integral y sostenible, siendo estas una relación con el contexto social, cultural y biofísico, el pensamiento de la vivienda como un lugar para el habitar que involucra una cualificación propia y una correspondencia con el ambiente y la forma en que el ámbito posibilita el crecientito humano (Salas et al., 2016). Por todo ello en el Ecuador los desafíos presentes en las ciudades y los asentamientos humanos obligan a los funcionarios a buscar soluciones transversales mediante una alta diversidad de actores. Las personas de estrato social bajo que no tienen disponibilidad de acceder a una vivienda, cuando no acuden a un alquiler en condiciones inestables (o después de hacerlo), procederán a encontrar su estabilidad en invasiones y asentamientos irregulares apropiándose de manera ilegal de terrenos y lotes para ahí emplazar sus casas y así alcanzar a tener "algo propio" y conformar su patrimonio familiar; no hay duda de que esa es la más adecuada lógica y necesidad de criterio de las personas más pobres, y las políticas públicas de vivienda no pueden (o no quieren) tomar medidas efectivas para brindar a las personas opciones alternativas (Báez, 2020).

El costo de la vivienda social es muy elevado en el Ecuador y en algunos países de Latinoamérica por lo que la clase social de bajos recursos económicos no puede acceder a estos programas habitacionales, es por ello por lo que la autoconstrucción se ha convertido en la única alternativa viable para obtener vivienda en este nivel social (Báez et al., 2019). 
Por todo ello en este artículo se recogen los principales criterios territoriales y urbanos aplicados al desarrollo urbano, para el desarrollo de indicadores de sostenibilidad de la vivienda social de la ciudad de Cuenca Ecuador.

\section{Marco teórico}

Para un adecuado desarrollo de este trabajo y de los indicadores de sostenibilidad enfocados a la vivienda social es de mucha importancia el entender los conceptos de los elementos que componen a estos utilitos.

\section{Sostenibilidad}

Para entender de forma correcta la definición de sostenibilidad se vuelve necesario analizar el concepto presentado en la Cumbre de la Tierra de 1992, Conferencia de la ONU que, iniciando por un punto de vista economicista, puntualiza al desarrollo sostenible como el que garantiza el desarrollo económico y el bienestar social sin colocar en peligro la preservación y conservación de los recursos naturales para las futuras generaciones (Madroñero \& Guzmán, 2018).

Por tal sentido comúnmente se ha entendido a la sostenibilidad como la secuela de la relación invariable de la dimensión económica, ambiental y la social, en la que se puede ver su interdependencia para detener o minimizar los aspectos negativos en la naturaleza (Rivera et al., 2017). Además, la sostenibilidad urbana es un enfoque relativamente nuevo que adhiere el cuidado de ecosistemas, participación social y crecimiento económico igualitario para dar una solución a los problemas sociales existentes y aumentar la calidad de vida de los humanos (Machín et al., 2017).

Al respecto los objetivos del Desarrollo Sostenible son una convocatoria por parte de las Naciones Unidas a todos los países del planeta para enfrentar los extensos desafíos a los que se enfrenta la humanidad y lograr que todos los seres humanos posean las mismas oportunidades y alcancen a llevar una vida mejor sin comprometer al planeta. Además de promover la innovación y construcciones resilientes desarrollando ciudades y comunidades capaces de producir y consumir de manera sostenible (Rivera et al., 2017).

\section{Arquitectura sostenible}

La arquitectura sostenible es un proceso de innovación en el cual se instituyen razonamientos de desarrollo sustentable como la disminución de gastos en los recursos ambientales utilizados, la disminución de la contaminación al aire, suelo y agua, una mejora del confort y de la calidad del interior del inmueble (Rodríguez et al., 2018). Por ello la arquitectura sostenible tiene como meta final aportar a mejorar la calidad de vida de los seres humanos mediante la sostenibilidad. Para conseguirlo, 
requiere en primer lugar ocupar técnicas y materia prima menos degradantes y con una durabilidad más alta (Acosta, 2019).

\section{Vivienda asequible}

Se define como una vivienda asequible a la vivienda que consume un $30 \%$ o menos de los ingresos totales familiares. Mediante esta definición se puede mencionar, que en los EE. UU millones de personas. no tienen vivienda asequible, siendo una cifra que cada día crece más (Acosta, 2019). Por otra parte, la arquitectura asequible está enfocada en diseñar y edificar viviendas de bajo costo y fácil adquisición sin el requerimiento de reducir las comodidades de los residentes. Este tipo de arquitectura no está enfocada en lo visual, sino en los requerimientos de la sociedad y en encontrar soluciones adecuadas para resolverlas (Peralta, 2020).

\section{Indicadores}

Se define como un indicador a un parámetro cualitativo o cuantitativo que puede ser estimado con correspondencia a un determinado criterio. Puede representar de manera objetiva, verificable y certera peculiaridades del medio ambiente o de los sistemas económicos y sociales asociados (García et al., 2019).

Además, se conoce que los propósitos de los indicadores de sostenibilidad son tres, el primero es consentir un examen sintético del desarrollo sostenible teniendo que estar todos los indicadores fundamentados en metodologías reconocidas y en datos legítimos (Peralta, 2020). El segundo propósito es servir de orientación siendo útiles para identificar alternativas de políticas, futuras decisiones administrativas más convenientes para lograr los resultados propuestos. Finalmente, el tercer propósito es la comunicación siendo los indicadores transparentes y sencillamente entendibles, que se resuman en herramientas comunicacionales pudiendo ser utilizados para la dirección de políticas en todos los niveles (Nacif, 2016).

\section{Marco referencial}

Con respecto a la construcción sostenible en Europa podemos encontrar el trabajo realizado por Elorrieta et al. (2016), quienes analizaron la incorporación de los principios de sostenibilidad en la planificación territorial de España, para lo cual realizaron un análisis de los planes y leyes de organización del territorio a nivel regional. Llegando a la conclusión de que un proceso de integración de la sostenibilidad a la planificación territorial a nivel regional es pausado y con resultados pobremente visibles.

De igual manera se puede encontrar el estudio de Ali et al. (2020) quienes buscan identificar las principales barreras de la construcción sostenible en los Estados Unidos. Encontrando cuatro barreras principales, las cuales son restricciones antes de la 
construcción, limitaciones administrativas, limitaciones legislativas y limitaciones financieras y de planificación, concluyéndolos como los desafíos más influyentes que afronta la industria para desarrollar la construcción sostenible.

Por otra parte, con respecto a la construcción sostenible en América Latina se puede hallar el trabajo publicado por Alvarado (2018), quien busco identificar los principales principios y nociones en los que se basan los modelos de Ciudades Sostenibles teniendo prioridad en exponer el contexto de América Latina, específicamente México. Para lo cual un abordaje exploratorio-descriptivo mediante una comparación de las principales ciudades sostenibles a nivel mundial, concluyendo que en la necesidad de desarrollar ambientes sostenibles es necesario acelerar los procesos de aprendizaje, construcción y recolección de capacidades ambientales.

Así mismo con un enfoque en Latinoamérica específicamente en Chile, se halla la investigación de Arnet \& Naranjo (2019), en la que se buscó diseñar una metodología nueva para la valoración sostenible que ayude a desarrollar un modelo urbano complejo y compacto, mediante la elaboración de indicadores cuantitativos y cualitativos. Concluyendo que el manejo de estándares sostenibles afines a un enfoque tradicional de la sostenibilidad disminuye la obtención de indicadores que aporten al desarrollo sostenible de las grandes ciudades.

De igual manera el trabajo de Valverde et al. (2017), quienes mediante una revisión bibliográfica de cuatro criterios de clasificación de certificaciones ambientales para edificaciones sostenibles enfocándose en el contexto geográfico y social, la influencia, ponderación, accesibilidad y evaluación del impacto ambiental, buscan evaluar la factibilidad de la propuesta de una Bogotá Sostenible. Para lo cual sugieren el desarrollo de un instrumento informático que favorezca la accesibilidad y ajuste la aprobación de los créditos para responder adecuadamente a los requerimientos del sector social y geográfico de Bogotá.

Por otro lado, enfocándonos en el desarrollo de indicadores de sostenibilidad encontramos el trabajo de Nacif (2016), quien mediante el diseño de un grupo de indicadores urbanos como herramientas de monitoreo dinámico y permanente busca analizar la de la ciudad de San Juan Argentina, concluyendo que para el adecuado desarrollo de estos indicadores se deben tener en cuenta tres aspectos fundamentales siendo estos el físico-espacial, sociocultural y ambiental.

Así mismo encontramos el trabajo de Márquez et al. (2019) quienes diseñaron un sistema de indicadores para evaluar la sostenibilidad de las comunidades rurales del Ecuador en el cerco de la Agenda 21 Local, Para lo cual hacen uso de una revisión bibliográfica, de modelos de gestión, métodos y varias herramientas para la puesta en práctica de la Agenda 21 Local, concluyendo que se debe tener muy en cuenta las necesidades básicas de las 
comunidades rurales, los problemas ambientales, culturales, sociales, económicos, políticos, legales y territoriales para un adecuado desarrollo de indicadores.

\section{Metodología}

Para el desarrollo de este trabajo se hará uso de un enfoque cualitativo de nivel exploratorio de diseño fundamentado y tipo bibliográfico, ya que se realizará una revisión y exploración de antecedentes e información obtenida de informes, libros, artículos científicos y estatutos, que permitan el diseño de un sistema de indicadores para evaluar los contextos de sustentabilidad de la ciudad de estudio.

\section{Desarrollo}

Como primer punto se realizó una revisión bibliófila a las principales guías y normativas internacionales y nacionales para el desarrollo de indicadores de sostenibilidad urbana, las cuales son:

\section{Leed V4.1 Ciudades y Comunidades: Planificar y Diseñar}

Este es un sistema de certificación mundial para el diseño, construcción y operación de edificaciones verdes de gran desempeño. En las últimas décadas, diversas versiones de LEED han estimulado el mercado mundial de la construcción verde hacia adelante de forma gradual, con cerca de 93,000 proyectos inscritos y certificados (USGBC, 2020).

\section{Sustainable Development Goal 11}

El objetivo 11 o también conocido como "ODS urbano" tiene como meta principal lograr que las ciudades y los asentamientos humanos sean más inclusivos, resiliente, seguros y sostenible, por lo que la comunidad internacional se mostró de acuerdo con el definir a la urbanización y el desarrollo de la ciudad como una fuerza transformadora para el progreso (UN-Habitat, 2016).

\section{ISO 37101 Sistemas de Gestión para el Desarrollo Sostenible en las Comunidades}

Esta normativa internacional especifica los requisitos y provee orientación acerca del establecimiento de un sistema de gestión para el desarrollo sostenible en las colectividades. El objetivo principal de esta normativa es ayudar a las comunidades a instituir un marco que les favorezca optimar su enfoque en sus aspectos económicos, sociales y ambientales. Los requerimientos de esta norma pueden ser aplicados en comunidades de sin importar su tamaño en sus esfuerzos de desarrollo sostenible encaminados a una infraestructura inteligente, mucho más eficiente y resiliente, y, en como último punto, ayudar a optimizar su bienestar general. Además, está norma afronta asuntos específicos de sostenibilidad como la educación, la gobernanza, la atención médica, la innovación, la interdependencia, la seguridad, la inclusividad, muchas más, y 
posee la flexibilidad de incluir otras cuestiones de sostenibilidad que puedan ser notables para la comunidad que la ejecuta (ISO, 2016).

NTE INEN-ISO 37120 Desarrollo Sostenible en Comunidades - Indicadores para servicios de la ciudad y calidad de vida (ISO 37120:2014, IDT)

Esta Normativa se origina gracias a las Normas Internacionales orientadas a desarrollar un enfoque holístico e integrado del progreso sostenible y resiliencia, por lo que los conjuntos de indicadores normalizados entregan una orientación uniforme de todo lo que se puede medir y de la manera en que se mide. Sin embargo, no entrega un juicio de valor, o rango para un valor numérico de los indicadores (NTE INEN-ISO, 2018).

La adecuación de esta norma no concede un estatus en esta dirección. Una comunidad o ciudad que cumplan con esta norma respectivamente al cálculo de indicadores de servicios y del nivel de vida únicamente puede requerir el desempeño a tal efecto.

\section{Diseño de los Indicadores}

A continuación, se presentan los indicadores de sostenibilidad para una vivienda asequible, los cuales se diseñaron con el objetivo de medir el nivel de comodidades con las que cuentan las viviendas, como también las características económicas de los ciudadanos ya que están garantizan si podrán acceder o no a una vivienda.

\section{Tabla 1}

Indicador de tasa de empleo

\begin{tabular}{|c|c|c|}
\hline Marco de Indicadores & \multicolumn{2}{|c|}{ Nivel Económico } \\
\hline Nombre Indicador: & \multicolumn{2}{|l|}{ Tasa de Empleo } \\
\hline Objetivo del indicador: & \multicolumn{2}{|c|}{$\begin{array}{l}\text { Mide el número de ciudadanos que se encuentran en una situación } \\
\text { laboral activa. }\end{array}$} \\
\hline Método de & \multirow{2}{*}{\multicolumn{2}{|c|}{$\frac{\text { Numero de cuidadanos con empleo }}{\text { tal de la poblacion en edad de trabaj }}$}} \\
\hline Evaluación del indicador: & & \\
\hline Unidad de medición: & Porcentaje & \\
\hline Valor optimo: & $75.00 \%$ & \\
\hline Radios de cobertura: & Descripción & Valor \\
\hline 1)Alto & $\begin{array}{l}\text { La ciudad presenta un alto índice de } \\
\text { empleo. }\end{array}$ & $>75 \%$ \\
\hline 2)Medio & $\begin{array}{l}\text { La ciudad presenta un índice } \\
\text { mediano de empleo. }\end{array}$ & $>50 \% \mathrm{y}>75 \%$ \\
\hline 3) Bajo & $\begin{array}{l}\text { La ciudad presenta un bajo índice } \\
\text { de empleo. }\end{array}$ & $<50 \%$ \\
\hline
\end{tabular}


En la tabla 1 se presenta la formula y parámetros para el cálculo del indicador del nivel de tasa de empleo de los ciudadanos de la ciudad de Cuenca- Ecuador.

De acuerdo con el Instituto Nacional de Estadística y Censos (INEC, 2021), en la ciudad de Cuenca 313.182 individuos se encuentran en edad de trabajar y un total de 183.688 ciudadanos poseen un empleo.

$$
\text { Tasa de empleo }=\frac{183.688}{313.182} * 100 \% \quad=\frac{183.688}{313.182} * 100 \% \quad=58.65 \%
$$

Gracias al resultado de este indicador se puede mencionar que la Ciudad de Cuenca se encuentra con una tase de empleo de nivel medio, probablemente por la crisis económica que atraviesa el país.

\section{Tabla 2}

\section{Indicador dotación de vehículo de recolección de residuos solidos}

\begin{tabular}{|c|c|c|}
\hline Marco de Indicadores & \multicolumn{2}{|l|}{ Nivel Ambiental } \\
\hline Nombre Indicador: & \multicolumn{2}{|c|}{ Dotación de vehículo de recolección de Residuos Solidos } \\
\hline Objetivo del indicador: & \multicolumn{2}{|c|}{$\begin{array}{l}\text { Medir la cantidad de vehículo de recolección de residuos sólidos en } \\
\text { relación con el número de habitantes. }\end{array}$} \\
\hline Método de & \multirow{2}{*}{\multicolumn{2}{|c|}{ Poblacion total }} \\
\hline Evaluación del indicador: & & \\
\hline Unidad de medición: & \multicolumn{2}{|l|}{ Habitantes por vehículo de recolección } \\
\hline Valor optimo: & \multicolumn{2}{|l|}{8000 hab. / vehículo de recolección } \\
\hline Radios de cobertura: & Descripción & Valor \\
\hline Dotación optima & $\begin{array}{l}\text { Existe una cantidad idónea de vehículo de } \\
\text { recolección para el número de habitantes. }\end{array}$ & $\begin{array}{l}<5000 \text { hab. / vehículo } \\
\text { de recolección }\end{array}$ \\
\hline Dotación buena & $\begin{array}{l}\text { Existe una cantidad buena de vehículo de } \\
\text { recolección para el número de habitantes. }\end{array}$ & $\begin{array}{c}5000 \text { a } 8000 \text { hab. / } \\
\text { vehículo de } \\
\text { recolección }\end{array}$ \\
\hline Dotación aceptable & $\begin{array}{l}\text { Existe una cantidad aceptable de vehículo de } \\
\text { recolección para el número de habitantes. }\end{array}$ & $\begin{array}{l}8000 \text { a } 16000 \text { hab. / } \\
\text { vehículo de } \\
\text { recolección }\end{array}$ \\
\hline Dotación mejorable & $\begin{array}{l}\text { Existe una cantidad mejorable de vehículo de } \\
\text { recolección para el número de habitantes. }\end{array}$ & $\begin{array}{c}16000 \text { a } 26000 \text { hab. / } \\
\text { vehículo de } \\
\text { recolección }\end{array}$ \\
\hline Dotación deficiente & $\begin{array}{l}\text { Existe una cantidad deficiente de vehículo de } \\
\text { recolección para el número de habitantes. }\end{array}$ & $\begin{array}{l}>20000 \text { hab. / c } \\
\text { vehículo de } \\
\text { recolección }\end{array}$ \\
\hline
\end{tabular}


En la tabla 2 se presenta la formula y parámetros para el cálculo del indicador del nivel Dotación de vehículos para recolección de Residuos Sólidos en la ciudad de CuencaEcuador.

De acuerdo con la EMAP (2021), para la recolección de los desechos sólidos en la ciudad de cuenca se encuentran habilitados 34 vehículos de carga posterior, 4 vehículos de carga frontal y 2 Camiones de apoyo al Reciclaje 2, lo que da un total de 42 vehículos de recolección (EMAC, 2021). Por otra parte, de acuerdo con la proyección poblacional del INEC (2021) la ciudad de Cuenca cuenta con 648171habitantes.

$$
\begin{aligned}
& \text { Dotacion de Vehiculos } \\
& =\frac{\text { Poblacion total }}{\text { Numero de vehiculos }}
\end{aligned}=\frac{648171}{42}=15432 \text { habitantes por vehiculo }
$$

Gracias al resultado de este indicador se puede mencionar que la Ciudad de Cuenca se encuentra con dotación aceptable de vehículos de recolección de basura, esto ayuda a dar un adecuado servicio a los hogares de la ciudad, evitando problemas de acumulación de basura.

\section{Tabla 3}

\begin{tabular}{|c|c|c|}
\hline Marco de Indicadores & \multicolumn{2}{|l|}{ Nivel Ambiental } \\
\hline Nombre Indicador: & \multicolumn{2}{|l|}{ Viviendas que cuentan con servicios básicos } \\
\hline Objetivo del indicador: & \multicolumn{2}{|c|}{$\begin{array}{l}\text { Medir el porcentaje de viviendas que tienen acceso a servicio de agua } \\
\text { potable. }\end{array}$} \\
\hline Método de & \multirow{2}{*}{\multicolumn{2}{|c|}{$V . S . A=\frac{\text { viviendas con servicio de agua potable }}{\text { Numero total de viviendas }} * 100$}} \\
\hline Evaluación del indicador: & & \\
\hline Valor optimo: & $100 \%$ & \\
\hline Radios de cobertura: & Descripción & Valor \\
\hline 1)Alto & $\begin{array}{l}\text { La ciudad presenta un alto índice de acceso a } \\
\text { agua potable. }\end{array}$ & $>75 \%$ \\
\hline 2)Medio & $\begin{array}{l}\text { La ciudad presenta un índice mediano de } \\
\text { acceso a agua potable. }\end{array}$ & $>50 \% \mathrm{y}>75 \%$ \\
\hline 3) Bajo & $\begin{array}{l}\text { La ciudad presenta un bajo índice de acceso a } \\
\text { agua potable. }\end{array}$ & $<50 \%$ \\
\hline
\end{tabular}

Indicador de Viviendas que cuentan con servicios de agua potable

En la tabla 3 se presenta la formula y parámetros para el cálculo del indicador del porcentaje de viviendas que cuentan con agua potable en la ciudad de Cuenca- Ecuador.

Con respecto a la información sobre el número de viviendas que existen en la ciudad de Cuenca, debemos tomar la información proporcionada por el último censo de población 
y vivienda realizado por el INEC (2010) en el cual se menciona que en la ciudad de Cuenca existen un total de 174.573 viviendas (particulares y colectivas) más los datos de viviendas edificadas desde el año 2011 hasta el año 2018 por parte del INEC tenemos un total de 221.864 viviendas. Además de acuerdo con el informe de agua saneamiento e Higiene por parte del INEC (2018) 153.45 viviendas cuentan con servicios de agua potable.

$$
\begin{array}{ll}
\begin{array}{l}
\text { V. } A \\
\text { viviendas con servicios de agua potable }
\end{array} & =\frac{194.986}{221.828} \\
\text { Numero total de viviendas } & * 100
\end{array}=87.9 \%
$$

De acuerdo con los resultados de este indicador más del 75\% de los hogares de la ciudad de Cuenca cuentan con acceso a servicios básicos, esto demuestra que existe un alto índice se asequibilidad a agua potable en las viviendas de la ciudad.

\section{Tabla 4}

\begin{tabular}{|c|c|c|}
\hline Marco de Indicadores & \multicolumn{2}{|l|}{ Nivel Ambiental } \\
\hline Nombre Indicador: & \multirow{2}{*}{\multicolumn{2}{|c|}{$\begin{array}{l}\text { Viviendas que cuentan con servicios básicos } \\
\text { Medir el porcentaje de viviendas que tienen acceso a servicio de luz } \\
\text { eléctrica. }\end{array}$}} \\
\hline Objetivo del indicador: & & \\
\hline $\begin{array}{l}\text { Método de } \\
\text { Evaluación del indicador: }\end{array}$ & \multicolumn{2}{|c|}{ V.S.E. $=\frac{\text { viviendas con servicio de luz electrica }}{\text { Numero total de viviendas }} * 100$} \\
\hline Valor optimo: & \multicolumn{2}{|l|}{$100 \%$} \\
\hline Radios de cobertura: & Descripción & Valor \\
\hline 1)Alto & $\begin{array}{l}\text { La ciudad presenta un alto índice de acceso a } \\
\text { servicio de luz eléctrica. }\end{array}$ & $>75 \%$ \\
\hline 2)Medio & $\begin{array}{l}\text { La ciudad presenta un índice mediano de } \\
\text { acceso a servicio de luz eléctrica. }\end{array}$ & $>50 \%$ y $>75 \%$ \\
\hline 3) Bajo & $\begin{array}{l}\text { La ciudad presenta un bajo índice de acceso a } \\
\text { servicios servicio de luz eléctrica. }\end{array}$ & $<50 \%$ \\
\hline \multicolumn{3}{|c|}{$\begin{array}{l}\text { En la tabla } 4 \text { se presenta la formula y parámetros para el cálculo del indicador del } \\
\text { porcentaje de viviendas que cuentan con servicio de luz en la ciudad de Cuenca- Ecuador. }\end{array}$} \\
\hline $\begin{array}{l}\text { De acuerdo con el Info } \\
\text { Ecuatoriano, (ARCONE }\end{array}$ & $\begin{array}{l}\text { dística Anual y Multi } \\
\text { la ciudad de Cuenca } e\end{array}$ & $\begin{array}{l}\text { Sector Eléctri } \\
\text { total de } 179.2\end{array}$ \\
\hline
\end{tabular}

Indicador de Viviendas que cuentan con servicios de electricidad 


$$
\begin{array}{cll}
\text { V.S.E. }=\frac{\text { viviendas con servicio de luz electrica }}{\text { Numero total de viviendas }} & \begin{array}{ll}
\frac{179.234}{221.828} \\
* 100
\end{array} & * 100
\end{array}=80.79 \%
$$

De acuerdo con los resultados de este indicador más del 75\% de los hogares de la ciudad de Cuenca cuentan con acceso a luz eléctrica, esto demuestra que existe un alto índice se asequibilidad a luz eléctrica en las viviendas de la ciudad.

\section{Tabla 5}

Indicador de Viviendas que cuentan con servicios de alcantarillado

\begin{tabular}{|c|c|c|}
\hline Marco de Indicadores & \multicolumn{2}{|l|}{ Nivel Ambiental } \\
\hline Nombre Indicador: & \multirow{2}{*}{\multicolumn{2}{|c|}{$\begin{array}{l}\text { Viviendas que cuentan con servicio de alcantarillado } \\
\text { Medir el porcentaje de viviendas que tienen acceso a servicio de } \\
\text { alcantarillado }\end{array}$}} \\
\hline Objetivo del indicador: & & \\
\hline $\begin{array}{c}\text { Método de } \\
\text { Evaluación del indicador: }\end{array}$ & \multirow{2}{*}{\multicolumn{2}{|c|}{ V.S.A. $=\frac{\text { viviendas con servicio de alcantarillado }}{\text { Numero total de viviendas }} * 100$}} \\
\hline Valor optimo: & & \\
\hline Radios de cobertura: & Descripción & Valor \\
\hline 1)Alto & $\begin{array}{l}\text { La ciudad presenta un alto índice de acceso a } \\
\text { servicio de alcantarillado. }\end{array}$ & $>75 \%$ \\
\hline 2)Medio & $\begin{array}{l}\text { La ciudad presenta un índice mediano de acceso } \\
\text { a servicio de alcantarillado. }\end{array}$ & $>50 \%$ y $>75 \%$ \\
\hline 3) Bajo & $\begin{array}{l}\text { La ciudad presenta un bajo índice de acceso a } \\
\text { servicio de alcantarillado. }\end{array}$ & $<50 \%$ \\
\hline
\end{tabular}

En la tabla 5 se presenta la formula y parámetros para el cálculo del indicador del porcentaje de viviendas que cuentan con servicio de alcantarillado en la ciudad de Cuenca- Ecuador.

De acuerdo con el informe de agua saneamiento e Higiene por parte del INEC (2018) 153.45 viviendas cuentan con servicios de agua potable.

$$
\begin{array}{ll}
\begin{array}{l}
\text { V.S. } \text { A. } \\
=\frac{\text { viviendas con servicio de alcantarillado }}{\text { Numero total de viviendas }} * 100
\end{array} & =\frac{128.485}{221.828}
\end{array} \quad \begin{aligned}
& =73,6 \%
\end{aligned}
$$

De acuerdo con los resultados de este indicador menos del $75 \%$ de los hogares de la ciudad de Cuenca cuentan con acceso a luz eléctrica, esto demuestra que existe un mediano índice se asequibilidad al servicio de alcantarillado en las viviendas de la ciudad. 


\section{Tabla 6}

Indicador de Gasto en servicios Básicos

\begin{tabular}{|c|c|c|}
\hline Marco de Indicadores & \multicolumn{2}{|l|}{ Nivel Económico } \\
\hline Nombre Indicador: & \multicolumn{2}{|l|}{ Gasto en servicios Básicos } \\
\hline Objetivo del indicador: & \multicolumn{2}{|c|}{ Medir el Gasto en servicios Básicos. } \\
\hline Método de & \multirow{2}{*}{\multicolumn{2}{|c|}{ G.S.B. $=\frac{(\text { gasto promedio agua }+ \text { luz }+ \text { conectivida })}{\text { Ningreso promedio de los habitantes de cuenca }} * 100$}} \\
\hline Evaluación del indicador: & & \\
\hline Valor optimo: & \multicolumn{2}{|l|}{$30 \%$} \\
\hline Radios de cobertura: & Descripción & Valor \\
\hline 1)Alto & $\begin{array}{l}\text { La ciudad presenta un alto índice de acceso a } \\
\text { servicio de conectividad. }\end{array}$ & $>30 \%$ \\
\hline 2)Medio & $\begin{array}{l}\text { La ciudad presenta un índice mediano de acceso } \\
\text { a servicio de conectividad. }\end{array}$ & $>20 \% \mathrm{y}>30 \%$ \\
\hline 3) Bajo & $\begin{array}{l}\text { La ciudad presenta un bajo índice de acceso a } \\
\text { servicio de conectividad. }\end{array}$ & $<20$ \\
\hline
\end{tabular}

En la tabla 6 se presenta la formula y parámetros para el cálculo del indicador del porcentaje de gastos en servicios por parte de las viviendas de la Cuenca- Ecuador.

De acuerdo con el estudio realizado por Baquero y Quesada (2016) el consumo promedio luz al mes por hogar en la ciudad de Cuenca es de $243,7 \mathrm{kWh}$, si a esto le multiplicamos el valor del kWh de 10.74 centavos nos da un valor promedio de 25.51 dólares al mes. Así mismo de acuerdo con Molina et al. (2016) el consumo promedio de agua al mes por hogar en Cuenca es de $24 \mathrm{~m} 3$, si a esto le multiplicamos por el precio del $\mathrm{m} 3$ que es de 0.68 centavos y le sumamos 3 dólares de recarga nos da un total de 19.50 dólares promedio que paga hogar por el consumo de agua en la ciudad de Cuenca. Además de acuerdo con el INEC los hogares de Ecuador gastan un promedio de 21,63 dólares al mes en telefonía fija y 15.74. Finalmente, de acuerdo con el INEC (2021), el salario promedio de los habitantes de la ciudad de Cuenca es de 318 dólares al mes.

$$
\begin{aligned}
& \begin{array}{l}
\text { G.S. } B \\
=\frac{(\text { gasto promedio agua }+ \text { luz }+ \text { conectivida })}{\text { Ningreso promedio de los habitantes de cuenca }}
\end{array} \quad \begin{array}{c}
* 100 \% \\
* 100
\end{array}
\end{aligned}
$$

De acuerdo con este indicador el gasto promedio del ingreso mensual de los ciudadanos de cuenca en servicios básicos para el hogar es de $25.89 \%$, este dato demuestra que la ciudad garantiza una vivienda asequible para sus ciudadanos. 


\section{Discusión}

El diseño de los indicadores de sostenibilidad de vivienda asequible se basó en el análisis del a cantidad de viviendas que cuentan con servicios básicos como agua potable, alcantarillado, luz eléctrica, servicio de recolección de desechos, de forma casi idéntica al trabajo realizado por Nacif (2016), en el que se propone el diseño de indicadores urbanos de sustentabilidad, para lo cual el autor se enfoca en las comodidades que deberían poseer los hogares como acceso a educación, salud, servicio de recolección de basura y servicios básicos.

Por otra parte, en este trabajo se decidió evaluar la accesibilidad a cada servicio básico por separado, tomado en cuenta el número de hogares que pueden acceder a dicho servicio con respecto al total de viviendas que existen en la ciudad de Cuenca, planteando valores adecuados sobre el $75 \%$, lo que demostró que en servicios como el agua potable y la luz eléctrica la ciudad posee un alto acceso para las familias, sin embargo al mismo tiempo demuestra que un $25 \%$ o menos no puede acceder a este tipo de servicios. Esta clase de diseño de indicadores es parecido al trabajo realizado por Márquez et al. (2019), trabajo en el que los atures buscan un tipo de indicadores que ayuden al mejoramiento de la calidad de vida de sus ciudadanos, por lo que se enfocaron demostrar la falta de servicios básicos en comunidades rurales del ecuador.

Además, al hablar de acceso a servicios básicos, es importante evaluar la situación económica de los ciudadanos por lo que se implementó un indicador que permita conocer el porcentaje de empleo de la Ciudad, en el cual se obtuvo que solo un $58.65 \%$ de la población en edad de trabajar tiene un empleo, esto repercute gravemente al momento de poder acceder a una vivienda asequible ya que $41.3 \%$ de la población no cuenta con una fuente de ingresos lo que no les permite el pago de servicios básicos, pagar un arriendo o poder acceder a un crédito para la compra de una vivienda.

Con respecto al servicio de recolección de basura, específicamente al número de vehículos de recolección con los que cuenta la ciudad, es adecuado ya que gracias el indicador se pudo determinar que existen 15432 habitantes por cada vehículo, lo que demuestra que la ciudad cuenta con una dotación aceptable lo que ayuda a mantener limpia la ciudad ayudando a combatir la contaminación y dando un paso hacia la sostenibilidad ambiental.

Se entiende por vivienda asequible a aquella provista de servicios básicos como agua, luz, eléctrica, alcantarillado y conectividad, en la que las familias no gasten más del $30 \%$ de sus ingresos en el gasto de estos servicios (Acosta, 2019). Por lo que, de acuerdo con los resultados del indicador de gastos en servicios básicos por parte de la población de la ciudad de Cuenca, en el que se obtuvo que solo un 25,89\% del ingreso promedio mensual se destina al pago de dichos servicios, esto debido a que el consumo de agua y luz por 
parte de los cuencanos se ha visto reducido gracias a las campañas de la Gobernación de la Ciudad. Por todo ello se puede mencionar que dicha ciudad si proporciona una asequibilidad en vivienda a su población cuando se trate de servicios de agua potable, luz eléctrica, alcantarillado y conectividad y está cada vez más cerca de la sostenibilidad urbana.

\section{Conclusiones}

- Se lograron diseñar indicadores de sostenibilidad urbana con énfasis en la vivienda asequible, los cuales estuvieron enfocados a en los tres elementos básicos de sostenibilidad economía, sociedad y ambiente, además de buscar garantizar que las viviendas cuenten con todas las comodidades para los residentes lo que las hace asequibles. Los indicadores propuestos en el presente trabajo pueden resultar ser herramientas útiles para la toma de decisiones en el contexto de diseño, ejecución y evaluación de políticas de sostenibilidad, siempre que se efectúe todos los procesos que en este trabajo se han mencionado y explicitar, de la forma más sencilla sin perder rigor técnico.

- Con respecto al diseño de los indicadores de nivel social, estos deben estar enfocados a identificar el nivel de servicios que el gobierno de Cuenca brinda a sus ciudadanos para garantizarles una vivienda asequible, por lo que se deben resaltar que el contar con comodidades como servicios policiales, bomberos, educación y salud en un radio adecuado, además de que las autoridades ayuden al financiamiento de los ciudadanos para acceder a una vivienda hace que la ciudad se encamine hacia la sostenibilidad.

- Es recomendable que, al momento de diseñar un sistema de indicadores de sostenibilidad urbana, sea pertinente investigar modelos sustentables ya aplicados en grandes cuidadas, los cuales serán una guía que permitan que los indicadores a desarrollarse sean viables, medibles y confiables, que consigan identificar una cantidad importante de variables requeridas para el desarrollo de la sostenibilidad urbana.

- Además, al hablar de comodidades es una obligación al tratarse de sostenibilidad que las viviendas cuenten con los servicios básicos (agua potable, luz eléctrica y conectividad) además de que las familias sean conscientes y responsables en el uso de los mismos ya que para ser consideradas viviendas asequibles las familias no deben gastar más del 30\% de sus ingresos totales en el pago de estos, entonces los indicadores ambiéntales de sostenibilidad en vivienda asequible están enfocados a medir el nivel de servicios básicos y servicios de recolección de desechos sólidos con los que cuentan las viviendas en la ciudad de Cuenca. 


\section{Agradecimientos}

El presente artículo es parte del trabajo de investigación y titulación del Programa de Maestría en Construcción con Mención en Administración de la Construcción Sustentable de la Universidad Católica de Cuenca, vinculados al Proyecto de Investigación: INDICADORES DE SOSTENIBILIDAD URBANA PARA LA CIUDAD DE CUENCA - ECUADOR, por ello agradecemos a todos y cada uno de los instructores pertenecientes a los grupos de investigación; Ciudad, Ambiente y Tecnología (CAT), y Sistemas embebidos y visión artificial en ciencias, Arquitectónicas, Agropecuarias, Ambientales y Automática (SEVA4CA), por los conocimientos e información brindados para la elaboración del trabajo.

\section{Referencias Bibliográficas}

Acosta, D. (2019). Arquitectura y construcción sostenibles: conceptos, problemas y estrategias. De arquitectura, 4(1), 14-23. Obtenido de https://www.researchgate.net/publication/50392823_Arquitectura_y_construccio n_sostenibles_Conceptos_Problemas_Y_Estrategias

Ali, K., Mostafa, N., \& Mohammadsoroush, T. (2020). Identifying the Key Barriers to Promote Sustainable Construction in the United States: A Principal Component Analysis. Sustainability, 12(5088), 1-21. https://www.researchgate.net/publication/342361284_Identifying_the_Key_Barr iers_to_Promote_Sustainable_Construction_in_the_United_States_A_Principal_ Component_Analysis

Alvarado, R. (2018). Ciudad inteligente y sostenible: hacia un modelo de innovación inclusiva de innovación inclusiva. PAAKAT: revista de tecnología y sociedad, 7(13), 1-17. http://www.scielo.org.mx/pdf/prts/v7n13/2007-3607-prts-7-1300002.pdf

Álvarez, A. M. (2016). Retos de América Latina: Agenda para el Desarrollo Sostenible y Negociaciones del siglo XXI. Problemas del Desarrollo. Revista Latinoamericana de Economía, 47(186), https://www.redalyc.org/pdf/118/11846179002.pdf

ARCONEL. (2016). Estadística anual y multianual del sector eléctrico ecuatoriano. Agencia de regulación y control de electricidad. https://www.regulacionelectrica.gob.ec/wpcontent/uploads/downloads/2017/08/Estad\%C3\%ADstica-anual-y-multianualsector-el\%C3\%A9ctrico-2016.pdf 
Arnet, V., \& Naranjo, E. (2019). Nueva sostenibilidad para los barrios de la gran metrópolis chilen. $\quad$ ESTOA, $18(9), \quad$ 91-100. https://publicaciones.ucuenca.edu.ec/ojs/index.php/estoa/article/view/2578/2386

Baez, A., Hernández, C., \& Magdalys, A. (2019). Evaluación de sostenibilidad en proyectos de desarrollo. Ingenierías USBMed, 10(1), 34-39. https://revistas.usb.edu.co/index.php/ingUSBmed

Báez, C. (2020). Medición de los Objetivos de Desarrollo Sostenible en la Unión Europea a través de indicadores compuestos. Documentos de Trabajo, 1(5), 1-28. https://www.fundacioncarolina.es/wpcontent/uploads/2020/05/DT_FC_Especial2.pdf

Baquero, M., \& Quesada, F. (2016). Eficiencia energética en el sector residencial de la Ciudad de Cuenca, Ecuador. MASKANA, 7(2), 147-165. https://publicaciones.ucuenca.edu.ec/ojs/index.php/maskana/article/view/1065

Elorrieta, B., Olcina, J., \& Sánchez, D. (2016). La sostenibilidad en la planificación territorial de escala regional en España: estudio de casos. Cuadernos Geográficos, 55(1), 149-175. https://www.redalyc.org/pdf/171/17146265006.pdf

EMAC. (2021). Recolección. Empresa Pública Municipal de Aseo de Cuenca. Obtenido de https://emac.gob.ec/servicios/recoleccion/

García, J., Cazallo, A., Barragán, C., Mercado, M., Olarte, L., \& Meza, V. (2019). Indicadores de Eficacia y Eficiencia en la gestión de procura de materiales en empresas del sector construcción del Departamento del Atlántico, Colombia. Revista Espacios, 40(22), 16-27. http://www.revistaespacios.com/a19v40n22/a19v40n22p16.pdf

INEC. (2010). Resultados del Censo 2010 de población y vivienda en el Ecuador. Instituto Nacional de Estadística y Censos. https://www.planificacion.gob.ec/wpcontent/uploads/downloads/2017/05/CENSO-AZUAY-CA\%c3\%91ARMORONA-SANTIAGO.pdf

INEC. (2018). Agua, saneamiento e higiene. Instituto Nacional de Estadística y Censos. https://www.google.com/search?q=inec\&rlz=1C1AVFC_enEC921EC921\&oq=I NEC\&aqs $=$ chrome.0.69i59j46i67i175i199i433j0i6712j0i433i512j69i61j69i6012. $865 \mathrm{j} 0 \mathrm{j} 7 \&$ sourceid $=$ chrome $\& \mathrm{ie}=\mathrm{UTF}-8$

INEC. (2021). Encuesta Nacional de Empleo, Desempleo y Subempleo. Instituto Nacional de Estadística y Censos. https://www.ecuadorencifras.gob.ec/documentos/webinec/EMPLEO/2021/Trimestre-enero-marzo-2021/Trimestral\%20eneromarzo\%202021_Mercado_Laboral.pdf 
ISO 37101. (2016). Sustainable development in communities - Management system for sustainable development - Requirements with guidance for use. http://taom.academy/upload/ISO\%2037101-2016\%20\%281\%29.pdf

Machín, F., Céspedes, S., Riverón, A., \& Fernández, E. (2017). Sostenibilidad, ingeniería y enseñanza de las ciencias básicas. Marco teórico conceptual. Revista $\begin{array}{llll}\text { Iberoamericana de } & \text { Educación, }\end{array}$ https://rieoei.org/historico/documentos/rie73a08.pdf

Madroñero, S., \& Guzmán, T. (2018). Desarrollo sostenible: Aplicabilidad y sus tendencias. Tecnología en Marcha, 31(3), 122-130. https://www.scielo.sa.cr/pdf/tem/v31n3/0379-3982-tem-31-03-122.pdf

Márquez, L., Vasallo, Y., Cuétara, L., \& Sablón, N. (2019). Sistema de indicadores para la sostenibilidad en comunidades rurales del Ecuador en el marco de la Agenda 21 Local. Revista Espacios, 40(18), 28-45. https://www.revistaespacios.com/a19v40n18/a19v40n18p28.pdf

Molina, E., Quesada, F., Calle, A. O., \& Orellana, D. (2016). Consumo sustentable de agua en viviendas de la ciudad de Cuenca. Ingenius, 3(20), 28-38. http://scielo.senescyt.gob.ec/pdf/ing/n20/1390-650X-ing-20-00028.pdf

Nacif, N. (2016). Diseño de indicadores urbanos de sustentabilidad. el caso del Gran San Juan en Argentina. Urbano, 1(34), 6-15. https://www.redalyc.org/pdf/198/19849706002.pdf

NTE INEN-ISO 37120. (2018). Desarrollo Sostenible en Comunidades indicadores para servicios de la ciudad y calidad de vida (ISO 37120:2014, IDT). https://www.normalizacion.gob.ec/buzon/normas/nte_inen_iso_37120.pdf

Peralta, J. J. (2020). Sostenibilidad urbana en el contexto Latinoamericano y en el europeo. Cuaderno de Investigación Urbanística, 12(131). Obtenido de https://dialnet.unirioja.es/descarga/articulo/7672623.pdf

Rivera, J., Alcántara, G., Blanco, N., \& Pascal, E. (2017). ¿Desarrollo sostenible o sustentable? La controversia de un concepto. Revista Posgrado y Sociedad, 15(1), 57-67. Obtenido de https://dialnet.unirioja.es/descarga/articulo/6039009.pdf

Rodríguez, L., Villadiego, K., Padilla, S., \& Osorio, H. (2018). Arquitectura y urbanismo sostenible en Colombia. Bitácora 28, 3(1), $19 \quad-26$. http://www.scielo.org.co/pdf/biut/v28n3/0124-7913-biut-28-03-19.pdf

Salas, L., López, J., Gómez, S., Franco, D., \& Martínez, E. (2016). Ciudades sostenibles y saludables: estrategias en busca de la calidad de vida. Revista Facultad Nacional 


\section{Conciencia}

de

Salud

Pública,

$34(1)$,

105-110.

https://www.redalyc.org/pdf/120/12043924012.pdf

UN-Habitat. (2016). Sustainable Development Goal 11 Make cities and human settlements inclusive, safe, resilient, and sustainable.

USGBC. (2020). LEED v4.1 Ciudades y comunidades: planificar y diseñar. U.S. Green Building Council.

Valverde, A., Chavarro, D., \& Álvarez, A. (2017). Una aproximación al sistema voluntario de certificación de edificios denominado "Bogotá Construcción Sostenible". Arquitectura y Urbanismo, 38(3), 71-85. https://www.redalyc.org/pdf/3768/376854676006.pdf

\section{Conflicto de intereses}

Los autores declaran que no existen conflicto de intereses en relación con el artículo presentado.

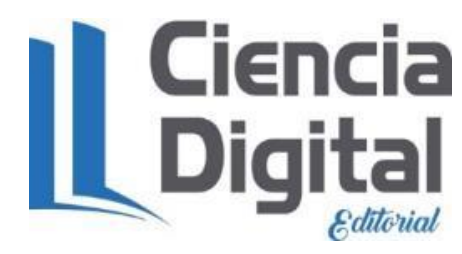




\section{Conciencia}

El artículo que se publica es de exclusiva responsabilidad de los autores y no necesariamente reflejan el pensamiento de la Revista Conciencia Digital.

\section{Liencia}

El artículo queda en propiedad de la revista y, por tanto, su publicación parcial y/o total en otro medio tiene que ser autorizado por el director de la Revista Conciencia Digital.
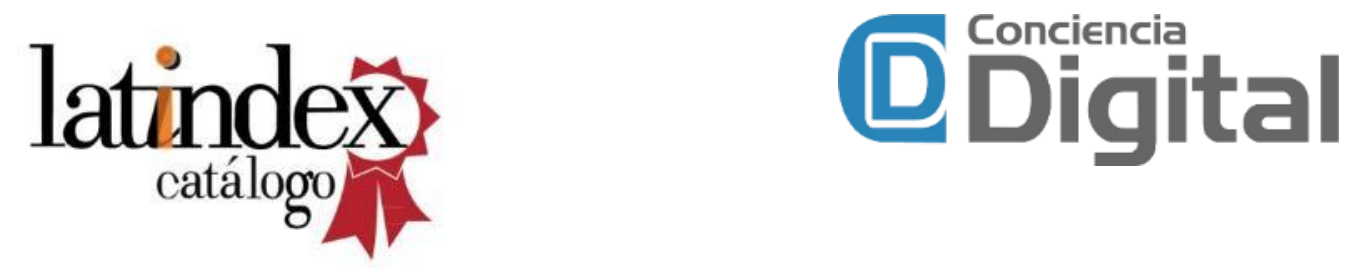

Indexaciones

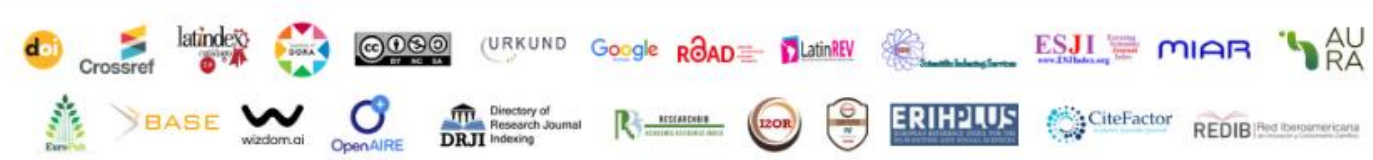

